\title{
Simulation of Stimulated Raman Scattering
}

\author{
Ahmad Naghidokht $\cdot$ Akbar Parvazian
}

Published online: 17 August 2012

(C) The Author(s) 2012. This article is published with open access at Springerlink.com

\begin{abstract}
Stimulated Raman scattering (SRS) with ordinary $(\mathrm{O}-$ mode) wave at phase space $(x, v)$ is investigated. For this purpose Vlasov equation is solved with one simulation method. Evolution of distribution function for an early time and a later time is presented. Initial distribution function has Gaussian shape, that is important in plasma heating, but as the time passed, this function due to the perturbation extends over space and velocity. In this situation, the behavior of distribution function has an important role in instability rate. Then instability rate for SRS is obtained and these results are showed in some special times and special cells. Density fluctuations affect instability rate and there is no remarkable damping at early times. At later times instability rate reduces sensitively which is in accordance to electron plasma wave damping and then heating the electrons. Also with increase in frequency of the incident wave, the instability rate due to saturation decreases sensitively.
\end{abstract}

Keywords Stimulated Raman scattering · Ordinary wave · Instability rate $\cdot$ Density fluctuations

\section{Introduction}

There is increasing experimental evidence that parametric instabilities especially stimulated Raman scattering (SRS) play an important role in laser induced fusion and also the radio frequency heating of magnetized plasmas [1-6].

A. Naghidokht $(\bowtie) \cdot$ A. Parvazian Department of Physics, Isfahan University of Technology, Isfahan, Iran e-mail: ahmadnaghidokht@gmail.com; ahmadnaghidokht@yahoo.com
The need for auxiliary heating in tokamaks, in addition to the Ohmic heating result from the toroidal currents, has been recognized since the early days of tokamak research. Apart from heating, noninductive current drive is also required for steady state operation [7]. In the electron cyclotron range of frequencies large-amplitude radio frequency waves have also been reported to be susceptible to parametric instabilities. In electron cyclotron resonance heating (ECRH) of a tokamak, one encounters anomalous absorption of a radio frequency wave. If the incident electromagnetic wave (pump wave) has a relatively long wavelength as compared with the wavelengths of the decay waves, then it is possible that energy is transferred to the particles via collective effects [8]. Controlling the growth rate of instabilities has long been recognized as a necessary condition for the feasibility of fusion schemes [9]. SRS is just one of these instabilities, whose critical feature is to generate energetic electrons, which can preheat the fusion plasmas. Although a lot of works has done about SRS for laser induced plasma but this phenomena occurs for magnetized plasma and especially in tokamaks too. An incident pump pulse is scattered by the electron density perturbation of a plasma wave in the stimulated Raman instability, while the plasma wave, in turn, arises from the decay of pump light wave. It is a resonant three-wave instability, which requires phase matching in both time and space that imposes frequency and wavenumber matching condition. In this paper we present our simulation results on the SRS with injection of an O-mode radio frequency wave with frequencies 28 and $58 \mathrm{GHz}$. Furthermore this work is done for wide range of frequencies from 28 to $138 \mathrm{GHz}$. We devoted "Simulation Method" section for explaining about simulation method. In "Evolution of Distribution Function" section we obtain the evolution of electron distribution function that may help us for recognition of the instability. Instability rate of SRS be 
studied in "Parameter Analysis" section. One subsection was devoted to application of SRS in spherical tokamaks. We discuss the results in "Discussion" section.

\section{Simulation Method}

The Vlasov equation is in the present paper advanced in time using the fourth-order Runge-Kutta method, and we wish to invoke the Maxwell equation into this scheme while still conserving the divergences of the electric and magnetic fields. This is performed by using the electrodynamic potentials together with the Lorentz condition, giving rise to Lorentz inhomogeneous wave equations. There are written in a form which ensures that the divergences of the electromagnetic fields are fulfilled up to the local truncation error of the numerical scheme, without requiring that the continuity equation for the currents and charges is fulfilled exactly. Together with the Fourier transform technique in velocity space, we are using pseudo-spectral methods for approximating derivations in space and fourth-order compact schemes to approximate derivatives in the Fourier transformed velocity space, and the standard fourth-order RungeKutta scheme to advance the system in time. We point out that the method is still restricted to periodic boundary conditions in space [10]. We discretise the problem on a rectangular, equidistant grid. The known variables in $\mathbf{x}$ space are discretised as $x_{1, i_{1}}=i_{1} \Delta x_{1}$ where $i_{1}=0,1, \ldots, N_{x_{1}}-1$ and $x_{2, i_{2}}=i_{2} \Delta x_{2}$ where $i_{2}=0,1, \ldots, N_{x_{2}}-1$. The grid sizes are $\Delta x_{1}=\frac{L_{1}}{N_{x_{1}}}$ and $\Delta x_{2}=\frac{L_{2}}{N_{x_{2}}}$ where $L_{1}$ and $L_{2}$ are the domain sizes in the $x_{1}$ and $x_{2}$ directions, respectively. We use the domain size $0 \leq \eta_{1} \leq \eta_{1, e, \max }$ and $0 \leq \eta_{2} \leq \eta_{2, e, \max }$ where $\eta$ is the Fourier transformed of the velocity variable $\mathbf{v}$. The known Fourier transformed velocity variable for electrons discretised as $\eta_{1, e, j_{1}}=j_{1} \Delta \eta_{1, e}$ where $j_{1}=0,1, \ldots, N_{\eta_{1}}$ and $\eta_{2, e, j_{2}}=j_{2} \Delta \eta_{2, e}$ where $j_{2}=-N_{\eta_{2}}, \ldots,-1,0,1, \ldots, N_{\eta_{2}}$. The grid sizes are $\Delta \eta_{1, e}=\frac{\eta_{1, e, \max }}{N_{\eta_{1}}}$ and $\Delta \eta_{2, e}=\frac{\eta_{2, e, \max }}{N_{\eta_{2}}}$. The time as discretised as $\mathrm{t}_{\mathrm{k}}=\mathrm{t}_{\mathrm{k}-1}+\Delta \mathrm{t}_{\mathrm{k}}$ where $\mathrm{k}=1,2, \ldots, N_{t}$ and the time step $\Delta t_{\mathrm{k}}$ is calculated adoptively. For ions also have same restrictions $[10,11]$.

\section{Evolution of Distribution Function}

The basic equation for description of collisionless plasma is Vlasov equation that can be written:

$\frac{\partial \mathrm{f}}{\partial \mathrm{t}}+\mathbf{v} \cdot \frac{\partial \mathrm{f}}{\partial \mathbf{r}}+\mathbf{a} \cdot \frac{\partial \mathrm{f}}{\partial \mathbf{v}}=0$

Vlasov equation explains fluctuation in distribution function with Fourier transformation in phase space. Following the injection of electromagnetic wave into plasma, fluctuations appear in the electron distribution function. We solved VlasovMaxwell equation at phase space $(x, v)$ and different times. Another cod about this is PIC (particle in cell) but our method solves Vlasov equation exactly but PIC involves with Poisson equation. The parameters used in the numerical simulation in this paper are as follows: the ion-electron mass ratio was $\frac{m_{i}}{m_{e}}=1836$, the speed of light to electron thermal velocity ratio was set to $\frac{\mathrm{c}}{\mathrm{V}_{\text {th }}}=22.59$, the ratio ion-electron temperature was set to $\frac{T_{i}}{T_{e}}=1.50$ and for the electron Vlasov equation $0 \leq$ $\eta_{1} \leq 10$ and $-10 \leq \eta_{2} \leq 10$. The number of intervals was set to $N_{x_{1}}=50, N_{\eta_{1}}=30$. We fixed other dimensions in this simulation, thus we have one dimension in space and one dimension in velocity. The number of time steps was $N_{\mathrm{t}}=$ 20,000. No numerical dissipation was used. We have $x_{1,0}=0$, $x_{1, \text { end }}=\frac{2 \pi}{\mathrm{k}_{x 1}}, x_{2,0}=0, x_{2, \text { end }}=\frac{2 \pi}{\mathrm{k}_{x 2}}$ where $\mathrm{k}_{x 1}=1.608682 \times$ $10^{-4}, \mathrm{k}_{x 2}=3.961163 \times 10^{-4}$. The initial condition for electrons was set to:

$\hat{\mathrm{f}}_{\mathrm{e}}\left(x_{1}, x_{2}, \eta_{1}, \eta_{2}, 0\right)=\mathrm{n}(x) \hat{\mathrm{f}}_{\mathrm{e}, 0}\left(\eta_{1}, \eta_{2}\right)$

$\hat{\mathrm{f}}_{\mathrm{e}, 0}\left(\eta_{1}, \eta_{2}\right)=\frac{1}{(2 \pi)^{2}} \exp \left[-\frac{1}{2}\left(\eta_{1}^{2}+\eta_{2}^{2}\right)\right]$

$\mathrm{n}(x)=\left[1+A \sum_{i_{1}}^{\frac{N_{x_{1}}}{2}-1} i_{1} \sin \left(i_{1} x_{1} k_{x 1}\right)\right]$

where $\mathrm{n}(x)$ is density perturbation and the initial amplitude of waves was $A=10^{-7}$. With this suggestions and simulation method that explained, we obtained evolution of electron distribution function. Figure 1 is initial distribution function that has Gaussian shape. In these calculations, distribution function, space and velocity normalized to average initial distribution function (according to $\mathrm{n}_{0} \simeq 10^{19} \mathrm{~m}^{-3}$ for NSTX), Debye length and thermal velocity, respectively.

Figure 2 is evolution of electron distribution function at an early time $\omega_{\text {pe }} \mathrm{t}=1200$ which shows initial disturbance. At a later time $\omega_{\mathrm{pe}} \mathrm{t}=20,000$ in Fig. 3, these disturbances extend over all space and all velocities.

\section{Parameter Analysis}

In most cases scattered wave due to instability is backscattered wave and forward scattered wave has not important role in instability rate. For coupling of wave modes into plasma resonance conditions for frequencies and wave numbers given by:

$\omega_{0}=\omega_{\mathrm{s}}+\omega$

$\mathbf{k}_{0}=\mathbf{k}_{\mathrm{s}}+\mathbf{k}$

where $\omega_{0}$ and $\omega_{\mathrm{s}}$ are frequencies of the incident and scattered wave in plasma, and $\omega$ is the frequency of the 


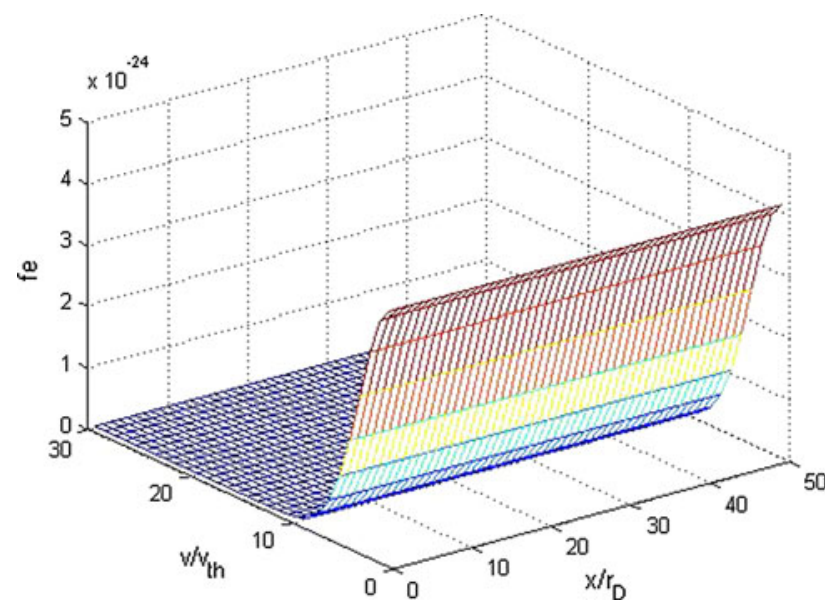

Fig. 1 Distribution function at time $\omega_{\text {pe }} \mathrm{t}=1$

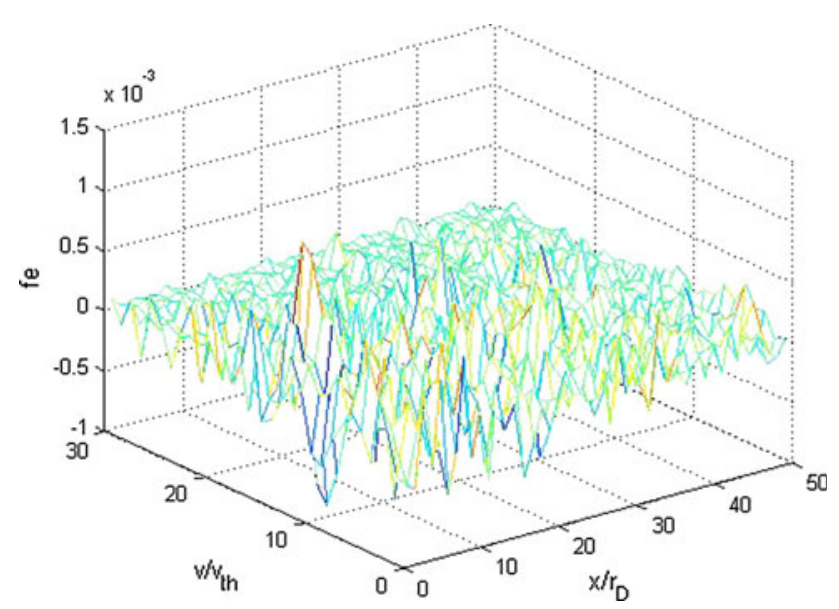

Fig. 2 Evolution of distribution function at time $\omega_{\mathrm{pe}} \mathrm{t}=1,200$

electron plasma wave (EPW). With considering of the dispersion relation of three waves that involved and coupling wave relations, we will have a nonlinear dispersion relation for resonant situations:

$\varepsilon_{\mathrm{s}}(\omega) \varepsilon\left(\omega-\omega_{0}\right)=\left(\lambda \frac{\mathrm{E}}{2}\right)^{2}$

where $\varepsilon_{j}(\omega)=\left(-\omega^{2}-2 i \omega \Gamma_{j}+\omega_{j}^{2}\right), \quad \Gamma_{j}$ is the linear damping for mode $j, \lambda=\frac{\omega_{\mathrm{p}} \mathrm{kq}_{\mathrm{e}}}{\mathrm{m}_{\mathrm{e}} \sqrt{\omega_{0}\left(\omega_{0}-\omega_{\mathrm{ek}}\right)}}$ and $\mathrm{E}$ is amplitude of the incident electric field. If we Solve this equation for SRS and suggest an imaginary part for frequency, $\omega=\omega_{\mathrm{r}}+i \gamma$, we will obtain an expression for instability rate of SRS that given by [12]:

$\gamma=\frac{\mathrm{kv}_{\mathrm{os}}}{4}\left(\frac{\omega_{\mathrm{pe}}^{2}}{\omega_{\mathrm{ek}}\left(\omega_{0}-\omega_{\mathrm{ek}}\right)}\right)^{\frac{1}{2}}$

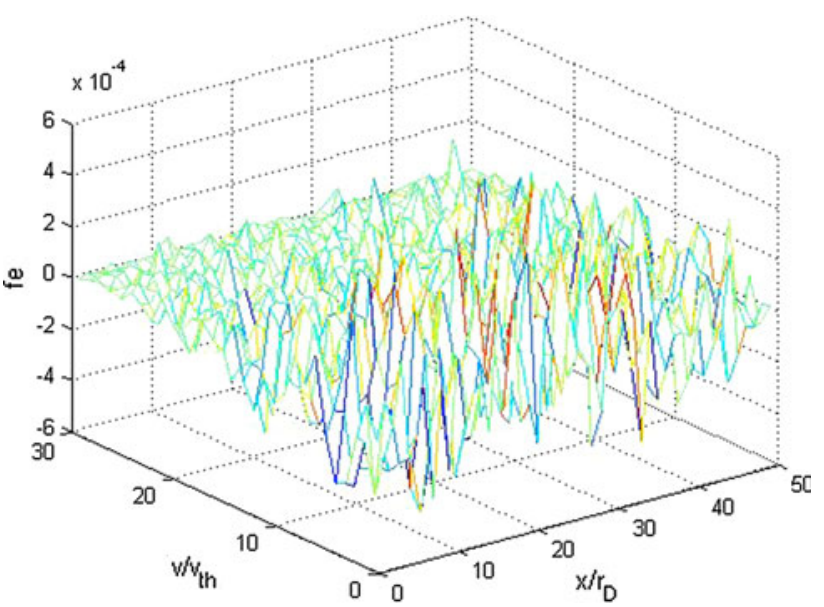

Fig. 3 Evolution of distribution function at time $\omega_{\mathrm{pe}} \mathrm{t}=20,000$

where $v_{\mathrm{os}}=\frac{\mathrm{q}_{\mathrm{e}} \mathrm{E}}{\mathrm{m}_{\mathrm{e}} \omega_{0}}$ is electron oscillating velocity, $\omega_{\mathrm{ek}}^{2}=$ $\omega_{\mathrm{pe}}^{2}+3 \mathrm{k}^{2} \mathrm{v}_{\mathrm{th}, \mathrm{e}}^{2}$ is the Bohm-Gross frequency for electron plasma wave and $v_{\mathrm{th}, \mathrm{e}}^{2}=\frac{2 \mathrm{kT}}{\mathrm{m}_{\mathrm{e}}}$ is square of electron thermal velocity.

\section{Application to Spherical Tokamaks}

In tokamaks the gyro-kinetic modeling is usually required to the treatment of the vlasov equation. For spherical tokamaks with low B (magnetic field), wave injection is applied but for tokamaks with high B this issue is not necessary because cyclotron frequency is high and itself leads to plasma heating. We used Vlasov equation instead of Boltzmann equation because we consider the situation of plasma heating by waves and Landau damping before any collision and interaction. Density fluctuation has an important role in instability rate i.e. the occurrence of electron density fluctuations which are localized in the phase-space has direct relationship to local maxima of the SRS instability. This issue is important for an efficient exploitation of the ECRH in tokamaks.

Frequency $\omega_{0}=28 \mathrm{GHz}$ is well known and applied frequency for heating of NSTX tokamak because optimum mode conversion to Bernstein waves, that have strong damping in electron cyclotron frequencies, occurs for this frequency [13]. We consider this frequency as an important issue in instability rate for this tokamak and in new point of view of SRS phenomena. Also frequency range $\omega_{0}=58-138 \mathrm{GHz}$ is applied frequency for MAST tokamak.

For the calculation, $\mathrm{kT}=300 \mathrm{eV}$ (k is Boltzmann constant here), $\mathrm{v}_{\mathrm{os}}=\mathrm{v}_{\mathrm{th}, \mathrm{e}} \sim 10^{6} \frac{\mathrm{m}}{\mathrm{s}}$ and $\mathrm{k}^{2} \lambda_{\mathrm{d}}^{2}=0.2$ be supposed. From simulation method that explained in previous section, we will also obtain electron density profile and therefore plasma frequency profile. Following them we will able to 


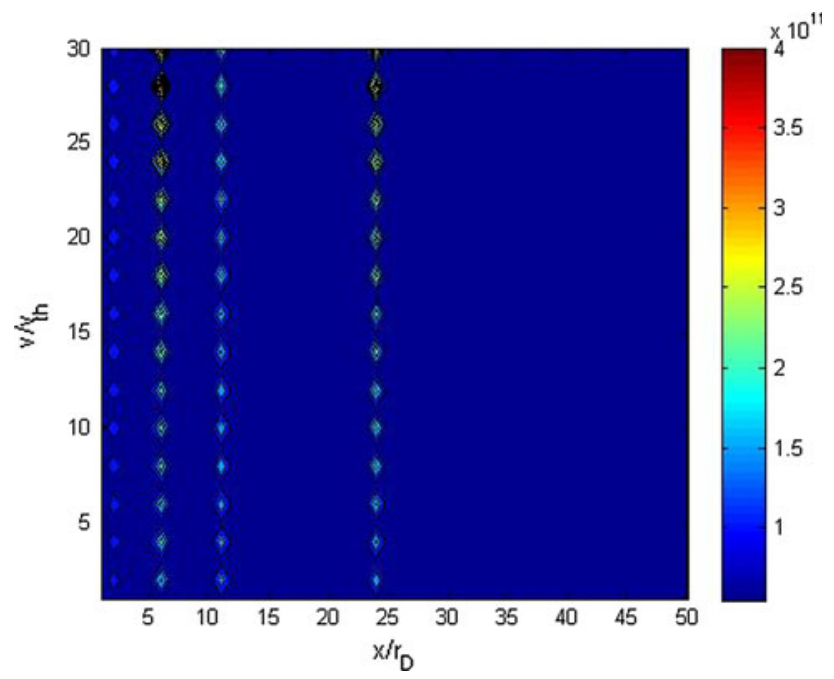

Fig. 4 Instability rate $\left(\gamma\left(\mathrm{s}^{-1}\right)\right)$ of SRS with $\omega_{0}=28 \mathrm{GHz}$ at time $\omega_{\text {pe }} \mathrm{t}=9,000$

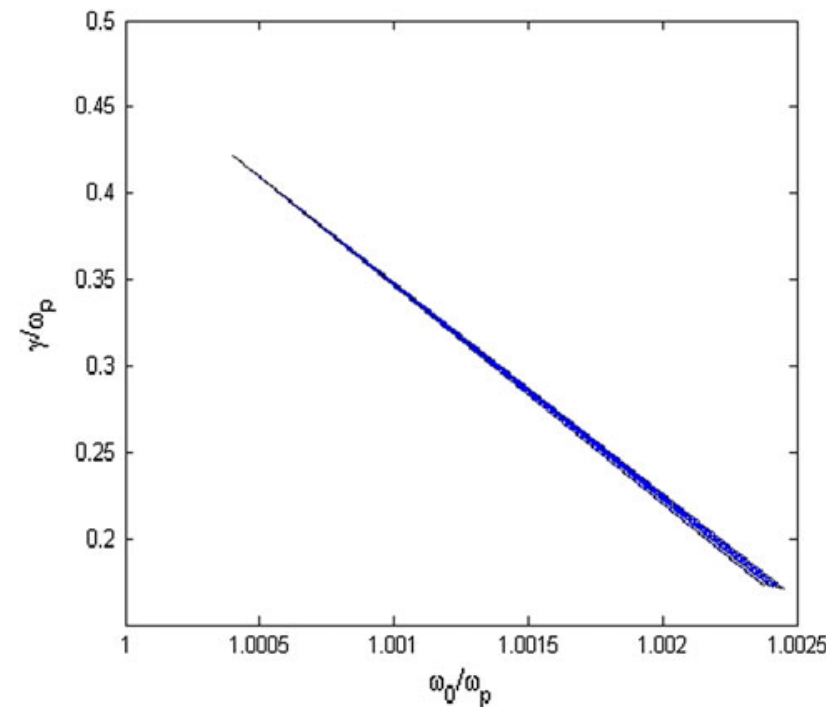

Fig. 5 Variation in normalized SRS instability rate $\frac{\gamma}{\omega_{\mathrm{p}}}$ with normalized pump frequency $\frac{\omega_{0}}{\omega_{\mathrm{p}}}$ with $\omega_{0}=28 \mathrm{GHz}$ for cell 46 and $\omega_{\text {pe }} \mathrm{t}=20,000$

calculate Debye length and from $\mathrm{k}^{2} \lambda_{\mathrm{d}}^{2}=0.2$, we will obtain a profile for wave number of the electron plasma wave.

At Fig. 4 instability rate with $\omega_{0}=28 \mathrm{GHz}$ at time $\omega_{\mathrm{pe}} \mathrm{t}=9,000$ shows damping effects of pump wave appeared in special cells. For cell 46 at later time, $\omega_{\mathrm{pe}} \mathrm{t}=20,000$, we have displayed normalized SRS instability rate $\frac{\gamma}{\omega_{\mathrm{p}}}$ as a function of $\frac{\omega_{0}}{\omega_{\mathrm{p}}}$ with $\omega_{0}=28 \mathrm{GHz}$ in Fig. 5 that goes as the square root of $\frac{\omega_{0}}{\omega_{\mathrm{p}}}$. The same behavior occurs for $\omega_{0}=58 \mathrm{GHz}$ frequency of the incident wave at equal later time $\omega_{\mathrm{pe}} \mathrm{t}=20,000$ for cell 46 , but instability rate is low by order two and this leads to reduction of wave

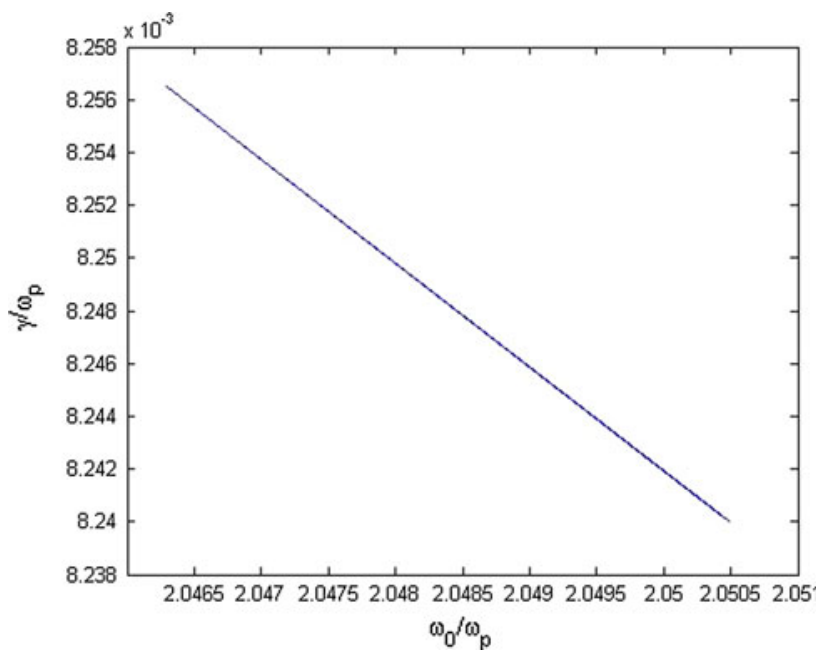

Fig. 6 Variation in normalized SRS instability rate $\frac{\gamma}{\omega_{\mathrm{p}}}$ with normalized pump frequency $\frac{\omega_{0}}{\omega_{\mathrm{p}}}$ with $\omega_{0}=58 \mathrm{GHz}$ and $\omega_{\mathrm{pe}} \mathrm{t}=20,000$ for cell 46
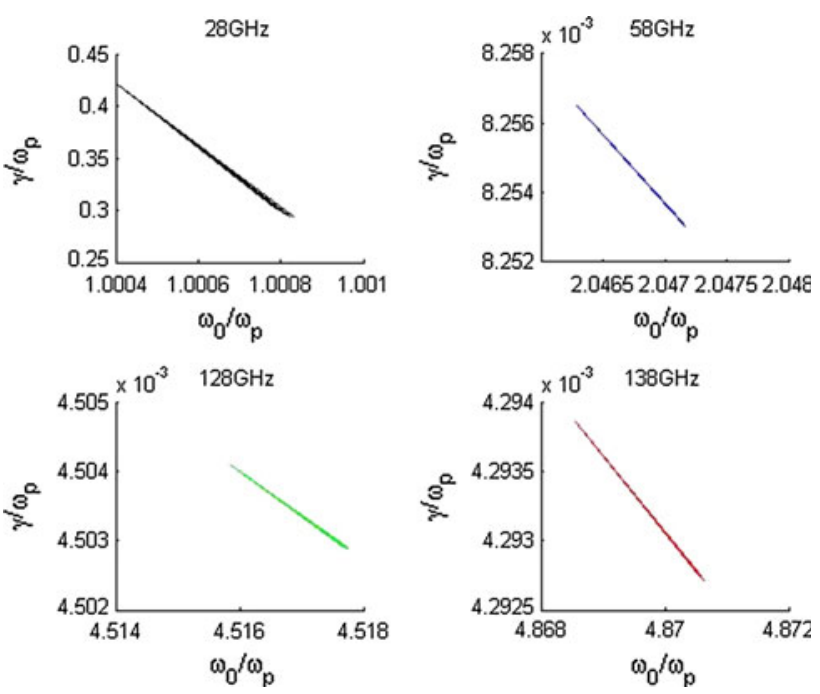

Fig. 7 Variation in normalized SRS instability rate $\frac{\gamma}{\omega_{\mathrm{p}}}$ with normalized pump frequency $\frac{\omega_{0}}{\omega_{\mathrm{p}}}$ with $\omega_{0}=28,58,128$ and $138 \mathrm{GHz}$ and $\omega_{\text {pe }} \mathrm{t}=10,000$ for cell 4

damping. This decrease of instability rate at higher frequencies of the incident radiation shows the three-wave instability to have already saturated. We presented these in Fig. 6. These figures show that as plasma frequency and following that density of plasma decreases, instability rate decreases too and vice versa.

We have displayed comparison between some frequencies and furthermore this calculation is done for continuous range of frequencies from 28 to $138 \mathrm{GHz}$. Results of these presented in Figs. 7 and 8.

Instability rate for $28 \mathrm{GHz}$ is high by order two compare to other three frequencies that shows high efficiency of this 


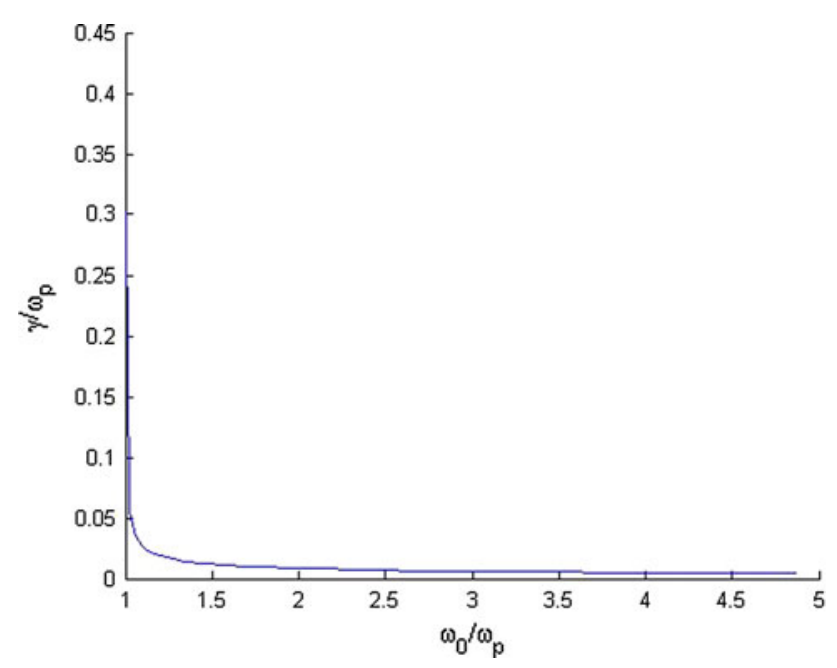

Fig. 8 Variation in normalized SRS instability rate $\frac{\gamma}{\omega_{\mathrm{p}}}$ with normalized pump frequency $\frac{\omega_{0}}{\omega_{\mathrm{p}}}$ with $\omega_{0}=28-138 \mathrm{GHz}$ and $\omega_{\mathrm{pe}} \mathrm{t}=10,000$ for cell 48

frequency for heating of NSTX tokamak which is in agreement with the results of Ram and Schultz [13] about applying $28 \mathrm{GHZ}$ as incident wave frequency into NSTX tokamak.

\section{Discussion}

The SRS instability provides an additional absorption mechanism by which the pump wave energy is transferred into electron plasma wave modes, eventually heating the electrons of plasma. As EPWs propagate out of the resonance region, where conditions for three waves resonant are not satisfied, they may suffer heavy cyclotron damping, heating the electrons. The enhanced transfer of energy from the high-frequency pump wave to the electrons is due to enhanced wave-particle interaction. The behavior of distribution function in different cells, different velocities and also different times has direct effect in the instability rate. Density fluctuations can affect wave modes coupling and then produce high oscillations in SRS instability rate. At later times, we can see reduction of SRS instability rate that leads to damping and then heating the electrons. Any increase in frequency of the injected wave decreases the instability rate, therefore as a result this shows that heating the electrons of plasma reduces.

Open Access This article is distributed under the terms of the Creative Commons Attribution License which permits any use, distribution, and reproduction in any medium, provided the original author(s) and the source are credited.

\section{References}

1. J.K. Kline, D.S. Montgomery, C. Rousseaux, S.D. Baton, V. Tassin, R.A. Hardin, K.A. Flippo, R.P. Johnson, T. Shimada, L. Yin et al., Laser Part. Beams 27, 185-190 (2009)

2. D.T. Michel, S. Depierrux, V. Tassin, C. Stenz, M. Chateau, C. Labaune, J. Phys. Conf. Ser. 244, 022022 (2010)

3. D. Benisti, O. Morice, L. Gremillet, E. Siminos, D.J. Strozzi, Phys. Rev. Lett. 105, 015001 (2010)

4. C. Rousseaux, S.D. Baton, D. Benisti, L. Gremillet, J.A. Adam, A. Heron, D.J. Strozzi, F. Amiranoff, Phys. Rev. Lett. 102, 185003 (2009)

5. L. Yin, B.J. Albright, K.J. Bowers, W. Daughton, H.A. Roase, Phys. Rev. Lett. 99, 265004 (2007)

6. A. Kumar, V.K. Tripathi, Phys. Plasmas 15, 062509 (2008)

7. R. Raman, D. Muller, B.A. Nelson, T.R. Jarboe, S. Gerhardt, H.W. Kugel, B. Leblance, R. Maingi, J. Menard, M. Ono et al., Phys. Rev. Lett. 104, 095003 (2010)

8. V.K. Tripathi, B.K. Sawhney, S.V. Singh, J. Plasma Phys. 50, 403 (1993)

9. C. Lihua, C. Tieqiang, L. Zhanjun, Z. Chunyang, Plasma Sci. Technol 9, 4 (2007)

10. B. Eliasson, J. Comput. Phys. 190, 501-522 (2003)

11. B. Eliasson, J. Comput. Phys. 181, 98-125 (2002)

12. W.L. Kruer, The Physics of Laser Plasma Interactions (AddisonWesley, Redwood City, 1988), p. 78

13. A.K. Ram, S.D. Schultz, PSFC/JA-00-24, 02139 (2000) 